\title{
Synthesis, antioxidant and $\alpha$-amylase inhibition activity of naphthalene-containing 2,4,5-trisubstituted imidazole derivatives
}

\author{
B SOMASHEKARA ${ }^{\mathrm{a}, \mathrm{c}}$, B THIPPESWAMY ${ }^{\mathrm{b}, \mathrm{c}}$ and G R VIJAYAKUMAR ${ }^{\mathrm{c}, *}$ \\ a Department of Chemistry, Smt. Indira Gandhi Government First Grade Women's College, Sagar, Karnataka \\ 577401 , India \\ ${ }^{b}$ Department of Chemistry, Government First Grade Science College, Chitradurga, Karnataka 577 501, India \\ ${ }^{\mathrm{c}}$ Department of Chemistry, University College of Science, Tumkur University, Tumkur, Karnataka 572 103, \\ India \\ E-mail: vijaykumargr18@gmail.com
}

MS received 8 February 2019; revised 26 April 2019; accepted 3 May 2019

\begin{abstract}
A series of naphthalene ring containing 2,4,5-trisubstituted imidazole derivatives (2a-2l) were synthesized using one-pot multicomponent reaction. The reactions were carried out using naphthaldehyde and substituted benzil in the presence of ammonium acetate in acetic acid media. All newly synthesized imidazole derivatives were characterized by FT-IR, ${ }^{1} \mathrm{H}$ NMR, ${ }^{13} \mathrm{C}$ NMR and mass spectral analysis. Newly synthesized imidazole derivatives were screened for their in-vitro antioxidant activity by DPPH free radical scavenging assay method and $\alpha$-amylase inhibition activity by DNS method. All the compounds showed excellent $\alpha$-amylase activity at 10,50 and $100 \mu \mathrm{g} / \mathrm{mL}$ and compounds $\mathbf{2 d}, \mathbf{2 g}, \mathbf{2 k}$ exhibited good antioxidant activity.
\end{abstract}

Keywords. 2,4,5-trisubstituted imidazole; one-pot multicomponent reaction; antioxidant activity; $\alpha$-amylase activity.

\section{Introduction}

In the recent years, imidazole derivatives received significant attention due to their biological and pharmacological importance. Many imidazole derivatives are occurring in natural compounds extracted from herbs. The substituted imidazole derivatives have a wide range of applications in pharmaceutical industries as an antioxidant, ${ }^{1}$ anticancer, ${ }^{2}$ anti-inflammatory, ${ }^{3}$ antimicrobial, ${ }^{4,5}$ antihypertension activity. ${ }^{6}$ On the other hand, the trisubstituted imidazole moieties are promising candidates for $\alpha$-amylase inhibition, ${ }^{7} \mathrm{C} 17,20$-lyase inhibition, ${ }^{8}$ glucosidase inhibition ${ }^{9,10}$ and kinase inhibition. ${ }^{11}$

The antioxidant property of the compound is very important to protect damage against free radicals that are produced in our body as a result of a biochemical reaction. Free radicals are generated due to internal factors such as inflammation and external factors such as environmental pollution, and UV exposure. These free radicals are dangers to the human body and which causes oxidative stress. Oxidative stress leads to cancer, ${ }^{12-14}$ heart diseases, ${ }^{15}$ osteoarthritis, ${ }^{16,17}$ stroke, ${ }^{18}$ respiratory diseases, ${ }^{19}$ immune deficiency, ${ }^{20}$ Parkinson's disease, ${ }^{21}$ emphysema ${ }^{22}$ and other inflammatory or ischemic conditions. Therefore, there is an urgent need to design and develop a highly active antioxidant compounds.

$\alpha$-Amylase inhibitors are the targets for the many researchers in drug-design for the development of a new class of compounds for the treatment of hyperlipaemia, diabetes and obesity. Enzyme inhibitors are the potential target in many diseases which control many important biochemical reactions. $\alpha$-Amylase inhibitors are the substances which control carbohydrate digestion and monosaccharide absorption. In this aspect, $\alpha$-amylase inhibitors are particularly important in maintaining a constant glucose level in the blood by delaying the breakdown of starch. Many available $\alpha$-amylase inhibitors are the microbial origin and their usage

\footnotetext{
*For correspondence

Electronic supplementary material: The online version of this article (https://doi.org/10.1007/s12039-019-1639-0) contains supplementary material, which is available to authorized users.
} 
has been limited due to their side effects. Various $\alpha$ amylase inhibitors are synthesized ${ }^{23,24}$ and screened for their inhibitory activity which include iminosugars, thiosugars, disaccharides, carbosugars and non-sugar derivatives. The $\alpha$-amylase inhibitors with a high degree of potency and specificity are still needed for the exploration of a new class of inhibitors. In this respect, several naphthalene incorporated heterocyclic compounds were synthesized and tested for their biological activities such as anticonvulsant, ${ }^{25,26}$ antimicrobial, ${ }^{27-29}$ antioxidant ${ }^{30}$ and anticancer activity. ${ }^{31,32}$ In view of the above observation, in our study we have synthesized naphthalene incorporated 2,4,5-trisubstituted imidazole derivatives and have screened for their antioxidant and $\alpha$-amylase inhibition activity.

\section{Experimental}

\subsection{Materials and methods}

Chemicals used in the present work were purchased from Sigma-Aldrich (India), Merck (Germany) and S. D. Fine Chemicals (India). All the laboratory grade solvents used were distilled before use. Melting points were determined by an open capillary method and were uncorrected. The IR spectra of the compounds ( $\mathrm{KBr}$ pellet method) were recorded on a JASCO FT/IR-4600 spectrophotometer. ${ }^{1} \mathrm{H}$ NMR and ${ }^{13} \mathrm{C}$ NMR spectra were recorded by using JEOL (500 MHz) NMR spectrometer and Agilent (400 MHz) NMR spectrometer respectively. The compounds were dissolved in either $\mathrm{DMSO}-d_{6}$ or $\mathrm{CDCl}_{3}$ for recording the spectra in the presence of TMS as an internal standard. Chemical shift values were presented in $\delta(\mathrm{ppm})$ scale. The mass spectra were recorded on a Agilent mass spectrometer instrument. The completion of the reactions was checked by thin layer chromatography (TLC) on silica gel coated aluminum sheets (silica gel 60 F254). The compounds name was given as per chem draw software. Antioxidant activity was carried out by DPPH free radical scavenging assay method. $\alpha$-Amylase inhibition activity by DNS method. The chemicals used for antioxidant and $\alpha$-amylase inhibition study was purchased from Hi-Media (Mumbai)-India. Absorbance was recorded using Systronics169 Visible Spectrophotometer.

\subsection{General procedure for the synthesis naphthalene incorporated 2,4,5-trisubstituted imidazoles (2a-2l)}

A mixture of naphthaldehyde (10 mmol), substituted benzil $(10 \mathrm{mmol})$ and ammonium acetate $(50 \mathrm{mmol})$ were dissolved in acetic acid in two necked $100 \mathrm{~mL}$ round bottom flask. The reaction mixture was refluxed for 12-14 h. The completion of reaction was monitored by TLC using petroleum ether and ethyl acetate (3:1) as eluent. After completion of the reaction, the mixture was poured into ice cold water. The solid compound precipitated was filtered and dried. All the crude products were purified by column chromatography except $\mathbf{2 a}$, $\mathbf{2 b}, \mathbf{2 c}, \mathbf{2 d}$ and $\mathbf{2 h}$, which purified by recrystallization using ethanol.

$2.2 \mathrm{a} \quad$ 4,5-bis(3-methoxylphenyl)-2-(naphthalen-2-yl)1H-imidazole (2a): Pale yellow solid; M.p. $184-186^{\circ} \mathrm{C}$; FT-IR $\left(\mathrm{KBr}, \mathrm{cm}^{-1}\right) v_{\max }: 3410.19(\mathrm{~N}-\mathrm{H}), 3051.80(\mathrm{Ar}-$ H) $1607.38(\mathrm{C}=\mathrm{C}), 1585.20(\mathrm{C}=\mathrm{N})$; ${ }^{1} \mathrm{H}$ NMR $(500 \mathrm{MHz}$, DMSO $\left.-d_{6}\right) \quad \delta(\mathrm{ppm}): 3.70\left(\mathrm{~s}, 3 \mathrm{H},-\mathrm{OCH}_{3}\right), 3.77(\mathrm{~s}, 3 \mathrm{H}$, $\left.-\mathrm{OCH}_{3}\right), 6.83(\mathrm{~m}, 1 \mathrm{H}, \mathrm{ArH}), 6.98(\mathrm{dd}, J=8.3 \mathrm{~Hz}, 1 \mathrm{H}$, ArH), $7.12(\mathrm{~m}, 2 \mathrm{H}, \mathrm{ArH}), 7.19$ (d, $J=7.6 \mathrm{~Hz}, 2 \mathrm{H}, \mathrm{ArH})$, $7.25(\mathrm{t}, J=7.9 \mathrm{~Hz}, 1 \mathrm{H}, \mathrm{ArH}), 7.38(\mathrm{t}, J=7.9 \mathrm{~Hz}, 1 \mathrm{H}$, ArH), $7.55(\mathrm{dd}, J=15.6 \mathrm{~Hz}, 6.5 \mathrm{~Hz}, 2 \mathrm{H}, \operatorname{ArH}), 7.96(\mathrm{q}$, $J=7.6 \mathrm{~Hz}, 2 \mathrm{H}, \mathrm{ArH}), 8.02(\mathrm{~d}, J=8.3 \mathrm{~Hz}, 1 \mathrm{H}, \mathrm{ArH}), 8.26$ (dd, $J=9.0 \mathrm{~Hz}, 1.4 \mathrm{~Hz}, 1 \mathrm{H}, \mathrm{ArH}), 8.61(\mathrm{~s}, 1 \mathrm{H}, \mathrm{ArH}), 12.85$ $(\mathrm{s}, 1 \mathrm{H}, \mathrm{NH}),{ }^{13} \mathrm{C}$ NMR $\left(400 \mathrm{MHz}, \mathrm{DMSO}-d_{6}\right) \delta(\mathrm{ppm})$ : 49.9, 50.2, 107.3, 107.7, 108.4, 109.0, 114.7, 115.9, 18.6, $118.8,121.4,121.7,122.8,122.9,123.1,123.3,123.6,124.2$, 124.8, 127.3, 127.8, 128.0, 131.5, 132.4, 140.4, 154.1, 154.3; MS $(m / z)$ : Calcd.406.16, found $407.17[\mathrm{M}+1]$.

$2.2 \mathrm{~b}$ 4,5-bis(3-methoxylphenyl)-2-(naphthalen-1-yl)$1 \mathrm{H}$-imidazole $(2 b)$ : Off white solid; M.p.: $185-186^{\circ} \mathrm{C}$; FT-IR $\left(\mathrm{KBr}, \mathrm{cm}^{-1}\right) v_{\max }: 3418.18(\mathrm{~N}-\mathrm{H}), 3049.80(\mathrm{Ar}-$ $\mathrm{H}), 1606.41(\mathrm{C}=\mathrm{C}), 1579.41(\mathrm{C}=\mathrm{N}) ;{ }^{1} \mathrm{H}$ NMR $(500 \mathrm{MHz}$, $\left.\mathrm{CDCl}_{3}\right) \quad \delta(\mathrm{ppm}): 3.77\left(\mathrm{~s}, 6 \mathrm{H},-\mathrm{OCH}_{3}\right), 6.83(\mathrm{dd}, J=8.3 \mathrm{~Hz}$, $2.1 \mathrm{~Hz}, 2 \mathrm{H}, \mathrm{ArH}), 6.90$ (dd, $J=8.3 \mathrm{~Hz}, 2.1 \mathrm{~Hz}, 1 \mathrm{H}, \mathrm{ArH})$, $7.06(\mathrm{~s}, 1 \mathrm{H}, \mathrm{ArH}), 7.11(\mathrm{~d}, J=7.6 \mathrm{~Hz}, 1 \mathrm{H}, \mathrm{ArH}), 7.23(\mathrm{~d}$, $J=8.3 \mathrm{~Hz}, 1 \mathrm{H}, \mathrm{ArH}), 7.34(\mathrm{q}, J=8.7 \mathrm{~Hz}, 3 \mathrm{H}, \mathrm{ArH}), 7.56$ (m, 2H, ArH), $7.78(\mathrm{dd}, J=6.9 \mathrm{~Hz}, 1.4 \mathrm{~Hz}, 1 \mathrm{H}, \mathrm{ArH}), 7.92$ (m, 2H, ArH), $8.82(\mathrm{~d}, J=8.3 \mathrm{~Hz}, 1 \mathrm{H}, \mathrm{ArH}), 9.33(\mathrm{~s}, 1 \mathrm{H}$, $\mathrm{NH}) ;{ }^{13} \mathrm{C}$ NMR $\left(400 \mathrm{MHz}, \mathrm{DMSO}-d_{6}\right), \delta(\mathrm{ppm}): 49.9,50.1$, 107.1, 107.9, 108.5, 108.8, 114.8, 115.7, 120.3, 121.2, 121.6, $121.7,121.8,122.5,122.9,123.3,124.0,124.3,124.7,125.4$, $127.2,128.7,131.7,132.1,140.5,154.2,154.3 ; \mathrm{MS}(\mathrm{m} / \mathrm{z})$ : Calcd.406.16, found $407.03[\mathrm{M}+1]$.

\section{2c 4,5-bis(4-methylphenyl)-2-(naphthalen-1-yl)-}

1H-imidazole (2c): Off white solid; M.p.: $280-284^{\circ} \mathrm{C}$; FT-IR $\left(\mathrm{KBr}, \mathrm{cm}^{-1}\right) v_{\max }: 3391.21(\mathrm{~N}-\mathrm{H}), 3063.37(\mathrm{Ar}-$ $\mathrm{H}), 1614.13(\mathrm{C}=\mathrm{C}), 1590.02(\mathrm{C}=\mathrm{N}) ;{ }^{1} \mathrm{H}$ NMR $(500 \mathrm{MHz}$, $\left.\mathrm{CDCl}_{3}\right) \quad \delta(\mathrm{ppm}): 2.36\left(\mathrm{~s}, 3 \mathrm{H},-\mathrm{CH}_{3}\right), 2.40\left(\mathrm{~S}, 3 \mathrm{H},-\mathrm{CH}_{3}\right)$, $7.15(\mathrm{~d}, J=8.3 \mathrm{~Hz}, 2 \mathrm{H}, \mathrm{ArH}), 7.22(\mathrm{~d}, J=7.6 \mathrm{~Hz}, 2 \mathrm{H}$, ArH), $7.42(\mathrm{~d}, J=7.6 \mathrm{~Hz}, 2 \mathrm{H}, \mathrm{ArH}), 7.56(\mathrm{~m}, 3 \mathrm{H}, \mathrm{ArH})$, $7.66(\mathrm{~d}, J=8.3 \mathrm{~Hz}, 2 \mathrm{H}, \mathrm{ArH}), 7.77(\mathrm{~m}, 1 \mathrm{H}, \mathrm{ArH}), 7.91(\mathrm{~m}$, $2 \mathrm{H}, \mathrm{ArH}), 8.84(\mathrm{~d}, J=8.3 \mathrm{~Hz}, 1 \mathrm{H}, \mathrm{ArH}), 9.22(\mathrm{~s}, 1 \mathrm{H}, \mathrm{NH})$; ${ }^{13} \mathrm{C}$ NMR (400 MHz, DMSO $\left.-d_{6}\right), \delta(\mathrm{ppm}): 21.2,125.6$, 126.5, 127.0, 127.5, 128.0, 128.5, 128.6, 129.6, 130.7, 133. $0,134.0,135.9,137.3,145.6$; MS $(m / z)$ : Calcd.374.17, found $373.17[\mathrm{M}-1]$.

\section{$2.2 \mathrm{~d}$ 4,5-bis(4-methylphenyl)-2-(naphthalen-2-yl)-}

$1 \mathrm{H}$-imidazole $(2 d)$ : $\quad$ Off white solid; M.p. $246-248^{\circ} \mathrm{C}$; FT-IR $\left(\mathrm{KBr}, \mathrm{cm}^{-1}\right) v_{\max }: 3418.21(\mathrm{~N}-\mathrm{H}), 3051.80(\mathrm{Ar}-\mathrm{H})$, $1614.13(\mathrm{C}=\mathrm{C}), 1589.06(\mathrm{C}=\mathrm{C}) ;{ }^{1} \mathrm{H}$ NMR $(500 \mathrm{MHz}$, DMSO $\left.-d_{6}\right) \quad \delta(\mathrm{ppm}): 2.30\left(\mathrm{~s}, 3 \mathrm{H},-\mathrm{CH}_{3}\right), 2.36(\mathrm{~s}, 3 \mathrm{H}$, 
$\left.-\mathrm{CH}_{3}\right), 7.13(\mathrm{~d}, J=7.6 \mathrm{~Hz}, 2 \mathrm{H}, \mathrm{ArH}), 7.26(\mathrm{~d}, J=8.3 \mathrm{~Hz}$, 2H, ArH), 7.45 (dd, $J=27.2,7.9 \mathrm{~Hz}, 4 \mathrm{H}, \mathrm{ArH}), 7.55(\mathrm{~m}$, 2H, ArH), 7.95 (dd, $J=13.4 \mathrm{~Hz}, 7.9 \mathrm{~Hz}, 2 \mathrm{H}, \mathrm{ArH}), 8.00$ $(\mathrm{d}, J=8.3 \mathrm{~Hz}, 1 \mathrm{H}, \mathrm{ArH}), 8.24(\mathrm{dd}, J=8.6 \mathrm{~Hz}, 1.7 \mathrm{~Hz}, 1 \mathrm{H}$, ArH), 8.60 (s, 1H, ArH), 12.74 (s, 1H, NH); ${ }^{13} \mathrm{C}$ NMR (400 $\left.\mathrm{MHz}, \mathrm{DMSO}-d_{6}\right), \delta(\mathrm{ppm}): 15.9,118.5,118.6,121.3,121.7$, 122.7, 122.9, 123.1, 123.2, 124.0, 127.7, 128.0, 131.4, 141.2; MS $(\mathrm{m} / \mathrm{z})$ : Calcd. 374.17 , found $375.05[\mathrm{M}+1]$.

2.2e 4,5-bis(4-bromophenyl)-2-(naphthalen-1-yl)-1H -imidazole (2e): Yellow solid; M.p.: $205-207^{\circ} \mathrm{C}$; FT-IR $\left(\mathrm{KBr}, \mathrm{cm}^{-1}\right) \nu_{\max }: 3417.28(\mathrm{~N}-\mathrm{H}), 1600.63(\mathrm{C}=\mathrm{C}), 1584.24$ $(\mathrm{C}=\mathrm{N}), 832 ;{ }^{1} \mathrm{H}$ NMR $\left(500 \mathrm{MHz}, \mathrm{CDCl}_{3}\right) \quad \delta(\mathrm{ppm}): 6.55(\mathrm{~m}$, $2 \mathrm{H}, \mathrm{ArH}), 6.64$ (d, $J=1.8 \mathrm{~Hz}, 2 \mathrm{H}, \mathrm{ArH}), 7.56$ (m, 4H, ArH), 7.64 (m, 2H, ArH), 7.6 (d, $J=3.4 \mathrm{~Hz}, 2 \mathrm{H}, \mathrm{ArH}), 7.78$ (d, $J=7.6 \mathrm{~Hz}, 2 \mathrm{H}, \mathrm{ArH}), 8.7(\mathrm{~d}, J=8.3 \mathrm{~Hz}, 1 \mathrm{H}, \mathrm{ArH}), 9.61$ $(\mathrm{s}, 1 \mathrm{H}, \mathrm{NH}) ;{ }^{13} \mathrm{C}$ NMR $\left(400 \mathrm{MHz}, \mathrm{DMSO}-d_{6}\right) \quad \delta(\mathrm{ppm})$ : 121.2, 121.8, 121.9, 124.3, 125.3, 126.4, 126.7, 126.8, 127.6; MS $(m / z)$ : Calcd.501.96, found 500.98 [M-1].

2.2f 2-(naphthalen-1-yl)-4,5-diphenyl-1H-imidazole (2f): Pale yellow solid; M.p.: $262-263^{\circ} \mathrm{C}$; FT-IR (KBr, $\left.\mathrm{cm}^{-1}\right) \nu_{\max }: 3417.24(\mathrm{~N}-\mathrm{H}), 3052.69$ (Ar-H), 1600.63 $(\mathrm{C}=\mathrm{C}), 1584.24(\mathrm{C}=\mathrm{N}) ;{ }^{1} \mathrm{H}$ NMR $\left(500 \mathrm{MHz}, \mathrm{DMSO}-d_{6}\right) \quad \delta$ (ppm): $7.25(\mathrm{t}, J=7.6 \mathrm{~Hz}, 1 \mathrm{H}, \mathrm{ArH}), 7.36(\mathrm{~m}, 3 \mathrm{H}, \mathrm{ArH})$, $7.45(\mathrm{t}, J=7.6 \mathrm{~Hz}, 2 \mathrm{H}, \mathrm{ArH}), 7.57(\mathrm{~m}, 2 \mathrm{H}, \mathrm{AH}), 7.62(\mathrm{~m}, 5 \mathrm{H}$, AH), $7.97(\mathrm{~d}, J=8.3 \mathrm{~Hz}, 1 \mathrm{H}, \mathrm{ArH}), 8.01(\mathrm{~d}, J=8.3 \mathrm{~Hz}$, 2H, ArH), 9.18 (d, $J=9.0 \mathrm{~Hz}, 1 \mathrm{H}, \mathrm{ArH}), 12.79$ (s, 1H, NH); ${ }^{13} \mathrm{C}$ NMR (400 MHz, DMSO $\left.-d_{6}\right), \delta(\mathrm{ppm}): 120.3,121.1$, 121.7, 121.8, 122.2 122.5, 122.7, 123.0, 123.3, 123.4, 123.7, $124.0,125.4,126.1,128.7,130.4,132.2,140.6$; MS $(m / z)$ : Calcd.346.14, found $347.02[\mathrm{M}+1]$.

$2.2 \mathrm{~g}$ 2-(naphthalen-2-yl)-4,5-diphenyl-1H-imidazole $(2 g)$ : $\quad$ Pale yellow solid; M.p.: $257-260{ }^{\circ} \mathrm{C}$; FT-IR $(\mathrm{KBr}$, $\left.\mathrm{cm}^{-1}\right) \nu_{\max }: 3417.24(\mathrm{~N}-\mathrm{H}), 3054.69$ (Ar-H), 1600.63 $(\mathrm{C}=\mathrm{C}), 1584.01(\mathrm{C}=\mathrm{N}) ;{ }^{1} \mathrm{H} \mathrm{NMR}\left(500 \mathrm{MHz}, \mathrm{DMSO}-d_{6}\right) \quad \delta$ (ppm): 7.25 (t, $J=7.6 \mathrm{~Hz}, 1 \mathrm{H}, \mathrm{ArH}), 7.33(\mathrm{t}, J=7.6 \mathrm{~Hz}$, 2H,ArH), $7.40(\mathrm{t}, J=7.2 \mathrm{~Hz}, 1 \mathrm{H}, \mathrm{ArH}), 7.47(\mathrm{t}, J=$ $7.6 \mathrm{~Hz}, 2 \mathrm{H}, \mathrm{ArH}), 7.55$ (q, $J=7.6 \mathrm{~Hz}, 4 \mathrm{H}, \mathrm{ArH}), 7.60$ $(\mathrm{d}, J=6.9 \mathrm{~Hz}, 2 \mathrm{H}, \mathrm{ArH}), 7.96(\mathrm{q}, J=7.1 \mathrm{~Hz}, 2 \mathrm{H}, \mathrm{ArH})$, $8.02(\mathrm{~d}, J=9.0 \mathrm{~Hz}, 1 \mathrm{H}, \mathrm{ArH}), 8.26(\mathrm{dd}, J=8.6 \mathrm{~Hz}, J=$ $1.7 \mathrm{~Hz}, 1 \mathrm{H}, \mathrm{ArH}), 8.62$ (s, 1H, ArH), 12.87 (s, $1 \mathrm{H}, \mathrm{NH}) ;{ }^{13} \mathrm{C}$ NMR (400 MHz, DMSO - $\left.d_{6}\right) \quad \delta(\mathrm{ppm}): 118.5,118.7,121.4$, $121.6,121.7,122.2,122.8,122.9,122.9,123.1,123.3,123.5$, $123.7,126.1,127.7,128.0,130.2,132.4,140.5 ; \mathrm{MS}(\mathrm{m} / \mathrm{z})$ : Calcd.346.14, found $347.03[\mathrm{M}+1]$.

2.2h 4,5-bis(4-methoxylphenyl)-2-(naphthalen-1-yl)1H-imidazole (2h): Off white solid; M.p.: $172-174{ }^{\circ} \mathrm{C}$; FT-IR (KBr, cm $\left.{ }^{-1}\right) \nu_{\max }$ : $3418.21(\mathrm{~N}-\mathrm{H}), 3046.98$ (Ar$\mathrm{H}), 1614.13(\mathrm{C}=\mathrm{C}), 1574.59(\mathrm{C}=\mathrm{N}) ;{ }^{1} \mathrm{H}$ NMR $(500 \mathrm{MHz}$, DMSO $\left.-d_{6}\right) \quad \delta(\mathrm{ppm}): 3.71\left(\mathrm{~s}, 3 \mathrm{H},-\mathrm{CH}_{3}\right), 3.80(\mathrm{~s}, 3 \mathrm{H}$, $\left.-\mathrm{CH}_{3}\right), 6.71(\mathrm{~d}, J=6.9 \mathrm{~Hz}, 1 \mathrm{H}, \mathrm{ArH}), 6.92(\mathrm{~m}, 4 \mathrm{H}, \mathrm{ArH})$, $7.41(\mathrm{t}, J=8.6 \mathrm{~Hz} 1 \mathrm{H}, \mathrm{ArH}), 7.61(\mathrm{~m}, 6 \mathrm{H}, \mathrm{ArH}), 7.79$ (d,
$J=8.3 \mathrm{~Hz}, 1 \mathrm{H}, \mathrm{ArH}), 7.99(\mathrm{t}, J=6.9 \mathrm{~Hz}, 2 \mathrm{H}, \mathrm{ArH}), 12.60$ $(\mathrm{s}, 1 \mathrm{H}, \mathrm{NH})$; MS $(\mathrm{m} / \mathrm{z})$ :Calcd.406.16, found 407.14 [M+1].

$2.2 \mathrm{i}$ 4-(4-chlorophenyl)-2-(napthalen-1-yl)-5-phenyl1H-imidazole ( $2 \mathrm{i}$ ): Off white solid; M.p. $255-257^{\circ} \mathrm{C}$; FT-IR $\left(\mathrm{KBr}, \mathrm{cm}^{-1}\right) \nu_{\max }: 3418.21(\mathrm{~N}-\mathrm{H}), 3049.87$ (Ar-H), $1599.66(\mathrm{C}=\mathrm{C}), 1583.27(\mathrm{C}=\mathrm{N}), 745.35(\mathrm{C}-\mathrm{Cl}) ;{ }^{1} \mathrm{H}$ NMR $\left(500 \mathrm{MHz}, \mathrm{DMSO}-d_{6}\right) \quad \delta(\mathrm{ppm}): 7.39(\mathrm{t}, J=7.2 \mathrm{~Hz}$, $1 \mathrm{H}, \mathrm{ArH}), 7.41$ (d, $J=7.6 \mathrm{~Hz}, 2 \mathrm{H}, \mathrm{ArH}), 7.49$ (m,2H, ArH), $7.57(\mathrm{~d}, J=6.9 \mathrm{~Hz}, 2 \mathrm{H}, \mathrm{ArH}), 7.63(\mathrm{~m}, 5 \mathrm{H}, \mathrm{ArH}), 7.96(\mathrm{~d}$, $J=7.6 \mathrm{~Hz}, 1 \mathrm{H}, \mathrm{ArH}), 8.01(\mathrm{~d}, J=7.6 \mathrm{~Hz}, 2 \mathrm{H}, \mathrm{ArH}), 9.14$ $(\mathrm{d}, J=8.3 \mathrm{~Hz}, 1 \mathrm{H}, \mathrm{ArH}), 12.85(\mathrm{~s}, 1 \mathrm{H}, \mathrm{NH}) ;{ }^{13} \mathrm{C}$ NMR $(400$ $\left.\mathrm{MHz}, \mathrm{DMSO}-d_{6}\right) \quad \delta(\mathrm{ppm}): 120.3,121.2,121.5,121.8$, $122.4,123.0,123.3,123.4,123.5,123.5,123.7,123.8,124.1$, 125.0, 125.4, 125.8, 126.0, 127.2, 128.7, 129.2, 130.1, 130.9, 132.7, 140.8, 140.9; MS ( $\mathrm{m} / \mathrm{z})$ : Calcd.380.10, found 381.14 $[\mathrm{M}+1]$.

2.2j 4-(4-chlorophenyl)-2-(napthalen-2-yl)-5-phenyl$1 \mathrm{H}$-imidazole (2j): $\quad$ Pale brown solid; M.p. $222-224{ }^{\circ} \mathrm{C}$; FT-IR (KBr, cm $\left.{ }^{-1}\right) \nu_{\max }: 3442.31(\mathrm{~N}-\mathrm{H}), 3056.62(\mathrm{Ar}-\mathrm{H})$, $1580.38(\mathrm{C}=\mathrm{N}), 753.06(\mathrm{C}-\mathrm{Cl}){ }^{1}{ }^{\mathrm{H}} \mathrm{NMR}(500 \mathrm{MHz}, \mathrm{DMSO}-$ $\left.d_{6}\right) \quad \delta(\mathrm{ppm}): 7.40(\mathrm{~m}, 4 \mathrm{H}, \mathrm{ArH}), 7.53(\mathrm{~m}, 7 \mathrm{H}, \mathrm{ArH}), 7.96(\mathrm{q}$, $J=7.6 \mathrm{~Hz}, 2 \mathrm{H}, \mathrm{ArH}), 8.02(\mathrm{~d}, J=9.0 \mathrm{~Hz}, 1 \mathrm{H}, \mathrm{ArH}), 8,25$ (dd, $J=8.6 \mathrm{~Hz}, 1.7 \mathrm{~Hz}, 1 \mathrm{H}, \mathrm{ArH}), 8.61$ (s,1H, ArH), 12.92 $(\mathrm{s}, 1 \mathrm{H}, \mathrm{NH}) ;{ }^{13} \mathrm{C}$ NMR $\left(400 \mathrm{MHz}, \mathrm{DMSO}-d_{6}\right) \quad \delta(\mathrm{ppm})$ : $118.5,118.8,121.4,121.8,122.7,122.8,123.2,123.3,123.6$, 127.8, 128.0, 140.8; MS ( $\mathrm{m} / \mathrm{z})$ :Calcd.380.10, found 380.98 $[\mathrm{M}+1]$.

2.2k 4,5-di(furan-2-yl)-2-(naphthalen-2-yl)-1H-imidazole (2k): Brown solid; M.p. $248-251^{\circ} \mathrm{C}$; FT-IR (KBr, $\left.\mathrm{cm}^{-1}\right) \quad v_{\max }: 3417.24(\mathrm{~N}-\mathrm{H}), 3057.58$ (Ar-H), 1601.59 $(\mathrm{C}=\mathrm{C}), 1555.31(\mathrm{C}=\mathrm{N}) ;{ }^{1} \mathrm{H}$ NMR $\left(500 \mathrm{MHz}, \mathrm{DMSO}-d_{6}\right) \quad \delta$ (ppm): $6.61(\mathrm{~d}, J=42.7 \mathrm{~Hz}, 2 \mathrm{H}, \mathrm{ArH}), 6.80(\mathrm{dd}, J=$ $100.0 \mathrm{~Hz}, J=2.8 \mathrm{~Hz}, 2 \mathrm{H}, \mathrm{ArH}), 7.57$ (m,2H, ArH), 7.81 $(\mathrm{d}, J=57.2 \mathrm{~Hz}, 2 \mathrm{H}, \mathrm{ArH}), 7.96(\mathrm{~d}, J=7.6 \mathrm{~Hz}, 1 \mathrm{H}, \mathrm{ArH})$, $8.01(\mathrm{t}, J=10.0 \mathrm{~Hz}, 2 \mathrm{H}, \operatorname{ArH}), 8.25(\mathrm{~d}, J=9.0 \mathrm{~Hz}, 1 \mathrm{H}$, ArH), 8.67 (s, 1H, ArH), 13.05 (s, 1H, NH); ${ }^{13} \mathrm{C}$ NMR (400 $\left.\mathrm{MHz}, \mathrm{DMSO}-d_{6}\right), \delta(\mathrm{ppm}): 102.0,103.5,106.4,107.0$, 114.7, 118.6, 119.4, 121.6, 121.8, 122.2, 122.7, 123.2, 123.3, $124.8,127.9,136.9,137.7,139.5,141.3,144.3$; MS $(m / z)$ : Calcd.326.10, found $327.11[\mathrm{M}+1]$.

\subsection{4-(3,4-dimethoxyphenyl)-5-(2-chlorophenyl)-2-}

(naphthalen-1-yl)-1H-imidazole (2l): Off white solid; M.p.: $185-187^{\circ} \mathrm{C}$; FT-IR $\left(\mathrm{KBr}, \mathrm{cm}^{-1}\right) \nu_{\max }: 3417.24(\mathrm{~N}-$ $\mathrm{H}), 3050.83(\mathrm{Ar}-\mathrm{H}), 1612.20(\mathrm{C}=\mathrm{C}), 1586.16(\mathrm{C}=\mathrm{N}), 786$ $(\mathrm{C}-\mathrm{Cl}) ;{ }^{1} \mathrm{H}$ NMR $\left(500 \mathrm{MHz}, \mathrm{DMSO}-d_{6}\right) \quad \delta(\mathrm{ppm}): 3.31$ $\left(\mathrm{s}, 3 \mathrm{H},-\mathrm{OCH}_{3}\right), 3.35\left(\mathrm{~s}, 3 \mathrm{H},-\mathrm{OCH}_{3}\right), 7.14(\mathrm{~d}, J=8.3 \mathrm{~Hz}$, $2 \mathrm{H}, \mathrm{ArH}), 7.25(\mathrm{~d}, J=7.6 \mathrm{~Hz}, 2 \mathrm{H}, \mathrm{ArH}), 7.44(\mathrm{~d}, J=$ $8.3 \mathrm{~Hz}, 2 \mathrm{H}, \mathrm{ArH}), 7.50(\mathrm{~d}, J=7.6 \mathrm{~Hz}, 2 \mathrm{H}, \mathrm{ArH}), 7.62(\mathrm{t}$, $J=7.6 \mathrm{~Hz}, 2 \mathrm{H}, \mathrm{ArH}), 7.94$ (d, $J=7.6 \mathrm{~Hz}, 1 \mathrm{H}, \mathrm{ArH}), 8.00$ (d, $J=7.6 \mathrm{~Hz}, 2 \mathrm{H}, \mathrm{ArH}), 9.16(\mathrm{~d}, J=9.0 \mathrm{~Hz}, 1 \mathrm{H}, \mathrm{ArH})$, $12.67(\mathrm{~s}, 1 \mathrm{H}, \mathrm{NH}) ;{ }^{13} \mathrm{C} \mathrm{NMR}\left(400 \mathrm{MHz}, \mathrm{DMSO}-d_{6}\right) \delta$ 
(ppm): 40.7, 50.0, 108.7, 109.1, 117.2, 118.0, 120.0, 120.3, 120.7, 121.1, 121.5, 121.7, 122.3, 122.6, 123.2, 123.5, 123.8, 124.1, 125.6, 126.9, 127.7, 127.9, 128.3, 131.9, 140.5, 152.9, 154.4; MS $(m / z)$ : Calcd.440.12, found $440.99[\mathrm{M}+1]$.

\subsection{Biological activity}

2.3a $\alpha$-Amylase inhibition assay: The $\alpha$-amylase inhibition assay was performed using the chromogenic DNS method reported by G. L. Miller. ${ }^{33}$ The total assay mixture composed of $1400 \mu \mathrm{L}$ of $0.05 \mathrm{M}$ sodium phosphate buffer (pH=6.9), $50 \mu \mathrm{L}$ of $\alpha$-amylase and samples at concentra- and reference Butylated hydroxy anisole (BHA) were taken in different test tubes. The volume was adjusted by adding $500 \mu \mathrm{L}$ of methanol. Five $\mathrm{mL}$ of $0.1 \mathrm{mM}$ methanolic solution of 1,1-diphenyl-2-picryl hydrazyl (DPPH) was added to the above test tubes and was shaken well for uniform concentration. A control without the test compounds, but with an equivalent amount of methanol was maintained. The test tubes were allowed to stand at room temperature for $20 \mathrm{~min}$ and absorbance of the samples was measured at $517 \mathrm{~nm}$. Free radical scavenging activity was calculated by using the formula. The experiments were conducted in triplicate and mean values are reported here.

$\%$ free radical scavenging $=\frac{(\text { Control OD }- \text { Sample OD })}{\text { Sample OD }} \times 100$

tion 10,50 and $100 \mu \mathrm{g} / \mathrm{mL}$ were incubated at $37^{\circ} \mathrm{C}$ for 10 $\mathrm{min}$. The reaction was terminated with $1 \mathrm{~mL}$ of DNS reagent, placed in boiling water bath for $5 \mathrm{~min}$, cooled to room temperature and the absorbance measured at $540 \mathrm{~nm}$. The control $\alpha$-amylase represented $100 \%$ enzyme activity and did not contain any samples of analysis. To eliminate the absorbance produced by sample appropriate extract controls with the extract in the reaction mixture in which the enzyme was added after adding DNS. The liberated sugar was determined by the help of standard maltose curve and activities were calculated according to the following formula.

\%inhibition/induction $=\frac{\text { Activity in presence of compound }}{\text { Control activity }} \times 100$

One unit of enzyme activity is defined as the amount of enzyme required to release one micromole of maltose from starch per minute under the assay conditions. The inhibitory/induction property shown by the sample was compared with that of control and expressed as percent induction/inhibition. This was calculated according to the following formula.

\section{Results and Discussion}

\subsection{Chemistry}

2,4,5-Trisubstituted imidazole derivatives (2a-2l) were synthesized by one-pot multicomponent reaction as shown in Scheme 1. The mechanism involves condensation followed by cyclization. In the cyclization process the water molecule is eliminated in acidic condition to reach the stable aromatic cyclic ring. The progress of

the reaction was monitored by Thin Layer Chromatography (TLC) using petroleum ether and ethyl acetate (3:1) medium and yield of the isolated compounds were recorded after the purification by column chromatography or recrystallization. Newly synthesized imidazole derivatives were characterized by melting point, FT-

Activity $=\frac{\text { Conc.of Maltose liberated } \mathrm{X} \mathrm{mL} \text { of enzyme used }}{\text { Mol.wt.of Maltose } \mathrm{X} \text { incubation time (min.) }} \mathrm{X}$ dilution factor

Acarbose was used as a standard inhibitor and it was assayed at above mentioned test sample concentrations. The assay method was similar to the above-mentioned procedure, instead of test samples, acarbose was added. The experiments were conducted in triplicate and results were compared to that of test samples.

\section{3b Antioxidant activity (Free radical scavenging} activities by DPPH assay): Free radical scavenging activity was performed using the DPPH assay method reported by Sunil Kumar et al. ${ }^{34}$ Different concentrations $(10 \mu \mathrm{g}, 50$ $\mu \mathrm{g}$ and $100 \mu \mathrm{g}$ ) of the samples were dissolved in DMSO
IR, NMR, and mass spectral analyses. Analytical and spectral data of the synthesized compounds were in full agreement with the proposed structures. In the IR spectra of the compounds, the presence of $\mathrm{NH}$ group of imidazole ring, aromatic $\mathrm{C}-\mathrm{H}, \mathrm{C}=\mathrm{N}$ and aromatic $\mathrm{C}=\mathrm{C}$ bonds have been clearly identified from their respective stretching frequencies. The number of proton signals in ${ }^{1} \mathrm{H}$ NMR spectrum and carbon signals in ${ }^{13} \mathrm{C}$ NMR spectrum were very much correlated with the respective compounds. Further, the compounds molecular mass was obtained from the mass spectra of the compounds. 
The complete characterization of the synthesized compounds has been given in the experimental section.

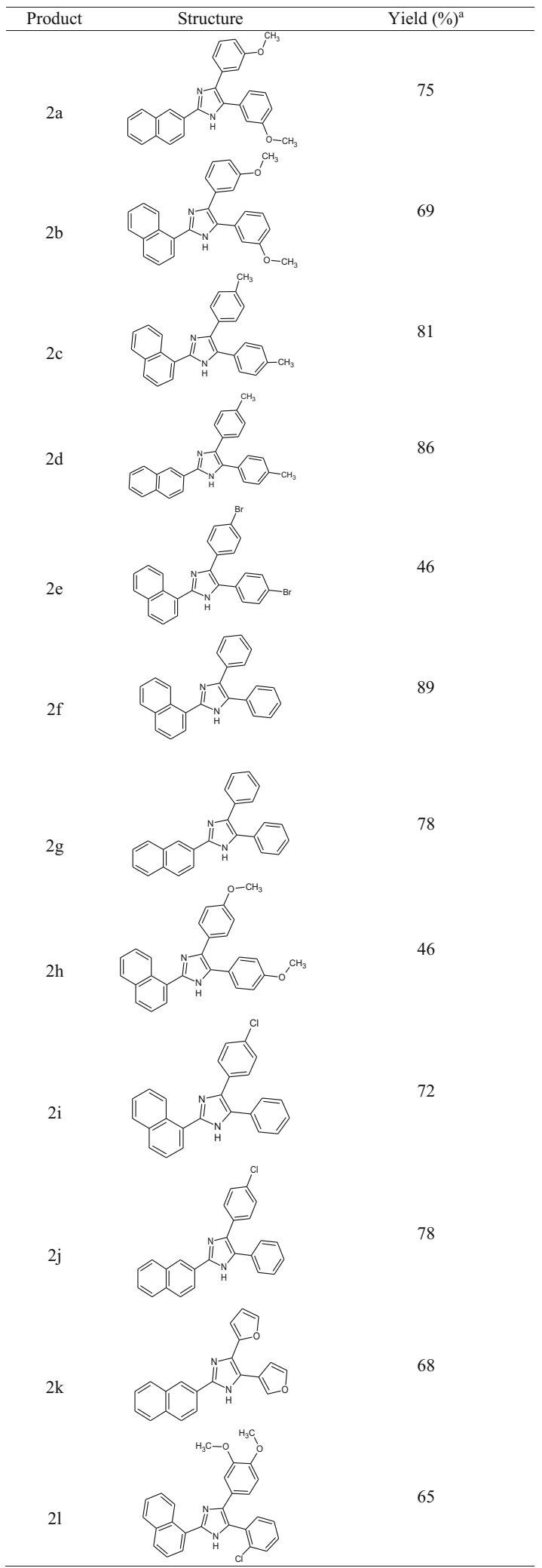

${ }^{\mathrm{a}}$ Isolated yield<smiles>[R]C=[O+]C([R])=O</smiles>

i: Ammonium acetate, acetic acid, $120^{\circ} \mathrm{C}, 12-14 \mathrm{~h}$.

Scheme 1. Synthesis of 2,4,5-trisubstituted imidazole derivatives (2a-2l).

\subsection{Biological results}

The $\alpha$-amylase inhibition activity revealed that all newly synthesized imidazole derivatives showed good inhibition at concentrations 10,50 and $100 \mu \mathrm{g} / \mathrm{mL}$. Among the prepared imidazole compounds $\mathbf{2 a}, \mathbf{2 b}, \mathbf{2 f}$ and $\mathbf{2 g}$ showed a bit higher inhibition compared to other compounds at $10 \mu \mathrm{g} / \mathrm{mL}$. The enhanced inhibition of 2,4,5-trisubstituted imidazole derivative compared with control enzyme might be due to the $\mathrm{N}-\mathrm{H}$ of imidazole core moiety and bit enhanced variations of inhibition might be due to the effect of functional groups attached to imidazole. The $\alpha$-amylases inhibition activity of the compounds (2a-2l) is shown in graphical representation (Figure 1).

Among the synthesized compounds, the compounds 2d, 2g, 2k exhibit good antioxidant activity at $100 \mu \mathrm{g} / \mathrm{mL}$ and other compounds exhibit moderate antioxidant activity as compared to the reference BHA. The enhanced activity of $\mathbf{2 d}, \mathbf{2} \mathbf{g}, \mathbf{2} \mathbf{k}$ imidazole derivatives may be due to 2-naphthyl substitution at second position and phenyl, 4-methyl-phenyl, furan substitution at 4,5 positions. The results of the antioxidant activity have been presented in Figure 2.

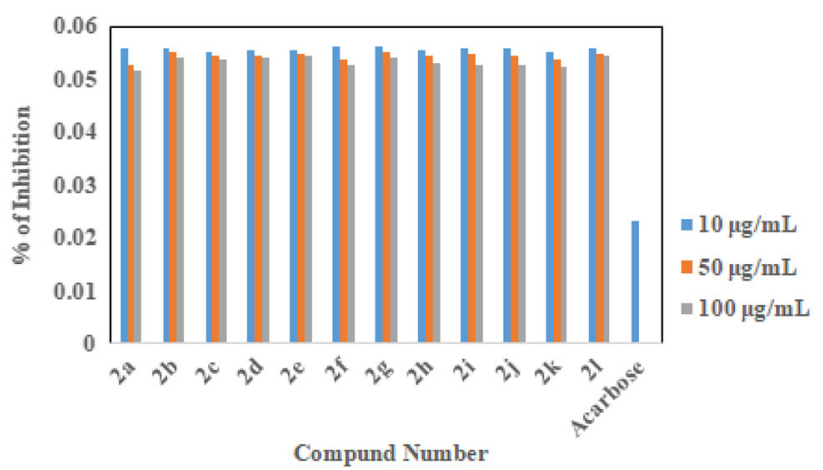

Figure 1. $\alpha$ - Amylase inhibition assay of 2,4,5-trisubstituted imidazole derivatives (2a-2I). 


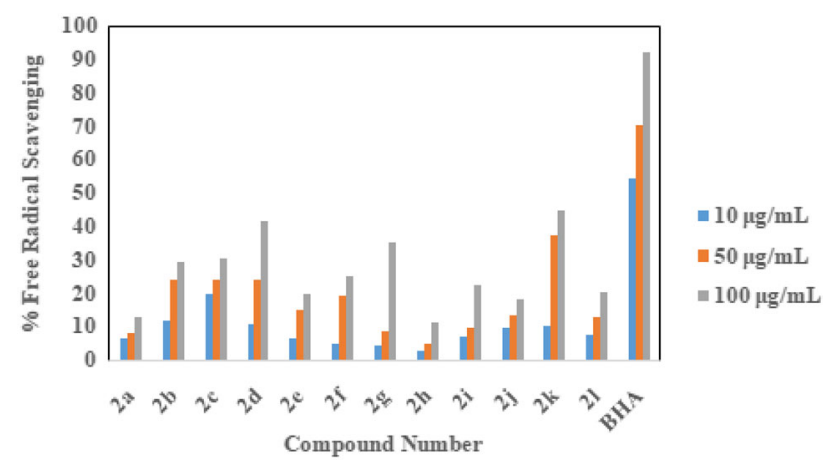

Figure 2. Antioxidant of 2,4,5-trisubstituted imidazole derivatives (2a-2l).

\section{Conclusions}

A series of naphthalene incorporated 2,4,5trisubstituted imidazole derivatives (2a-2l) were synthesized by one-pot multicomponent reaction and characterized by FT-IR, NMR and mass spectral analysis. The purified final compounds were investigated for their $\alpha$-amylase inhibition and antioxidant activities. All the compounds showed excellent $\alpha$-amylase inhibition as compared with the control sample. Among the screened samples, $\mathbf{2 d}, \mathbf{2 g}, \mathbf{2 k}$ exhibit good antioxidant activity compared to BHA standard. From this study, we conclude that the preparation of 2,4,5-trisubstituted imidazole using substituted benzil, naphthaldehyde, ammonium acetate and acetic acid media is the simplest method to construct imidazole ring. The biological study of the title compounds showed promising antioxidant and $\alpha$-amylase inhibition activities. Hence, they are ideally suited for further notification to obtain more efficient antioxidant and $\alpha$-amylase inhibition compounds.

\section{Supplementary Information (SI)}

${ }^{1} \mathrm{H},{ }^{13} \mathrm{C}$ NMR and Mass spectra of synthesized compounds are available at www.ias.ac.in/chemsci.

\section{Acknowledgements}

The authors are thankful to the Principal and the staff of Smt. Indira Gandhi Government First Grade Women's College, Sagar, Principal, University College of Science, Tumkur and Tumkur University administration for their support and encouragement.

\section{References}

1. Singh P, Kumar R, Tiwari S, Khanna R S, Tewari A K and Khanna H D 2015 Docking, synthesis and evaluation of antioxidant activity of 2,4,5-triaryl imidazole Clin. Med. Biochem. 11
2. Satyanarayana V S V, Rakshit M and Sivakumar A 2011 Microwave-assisted synthesis of 2,4,5-triphenyl$1 \mathrm{H}$-imidazole containing Schiff base derivatives with potential antioxidant and anticancer activities Asian $J$. Chem. 231212

3. Lombardino J G and Wiseman E H 1974 Preparation and anti-inflammatory activity of some nonacidic trisubstituted imidazoles J. Med. Chem. 171182

4. Sarala L, Merlin J P and Elanthamilan E 2016 Catalyst free synthesis of imidazoles: Characterization and its antimicrobial activity J. Chem. Pharm. Res. 8 225

5. Khan M S, Siddiqui S A, Siddiqui M S R A, Goswami U, Srinivasn K V and Khan M I 2008 Antibacterial activity of synthesized 2,4,5-trisubstituted imidazole derivatives Chem. Biol. Drug Des. 72197

6. Moutevelis-Minakakis P, Gianni M, Stougiannou H, Zoumpoulakis P, Zoga A, Vlahakos A D, Elliodromitis and Mavromoustakos T 2003 Design and synthesis of novel antihypertensive drugs Bioorg. Med. Chem. Lett. 131737

7. Balba M, El-Hady N A, Taha N, Rezki N and Ashry E S H E 2011 Inhibition of $\alpha$-glucosidase and $\alpha$-amylase by diaryl derivatives of imidazole-thione and 1,2,4-triazolethiol Eur. J. Med. Chem. 462596

8. Matsunaga N, Kaku T, Ojida A, Tanaka T, Hara T, Kusaka M and Tasaka A 2004 C17,20-lyase inhibitors. Part 2: Design, synthesis and structure-activity relationships of (2-naphthylmethyl)-1H-imidazoles as novel C17,20-lyase inhibitors Bioorg. Med. Chem. 124313

9. Naureen S, Noreen S, Nazeer A, Ashraf M, Alam U, Munawar M A and Khan M A 2015 Triarylimidazolessynthesis of 3-(4,5-diaryl-1H-imidazol-2-yl)-2-phenyl$1 \mathrm{H}$-indole derivatives as potent $\alpha$-glucosidase inhibitors Med. Chem. Res. 241586

10. Yar M, Bajda M, Shahzad S, Ullah N, Gilani M A, Ashraf M, Rauf A and Shaukat A 2015 Organocatalyzed solvent free an efficient novel synthesis of 2,4,5-trisubstituted imidazoles for $\alpha$-glucosidase inhibition to treat diabetes Bioorg. Chem. $\mathbf{5 8} 65$

11. Adams J L, Boehm J C, Gallagher T F, Shoukikassis, Hall E F W R, Sorenson M, Garigipati R, Griswold D E and Lee J C 2001 Pyrimidinylimidazole inhibitors of p38: Cyclic N-1 imidazole substituents enhance p38 kinase inhibition and oral activity Bioorg. Med. Chem. Lett. 11 2867

12. Sarshar S, Zhang C, Moran E J, Krane S, Rhodarte J C, Benbatoul K D, Dixon R and Mjali A M 2000 2,4,5Trisubstituted imidazoles: Novel nontoxic modulators of P-glycoprotein mediated multidrug resistance. Part 1 Bioorg. Med. Chem. Lett. 102599

13. Valkco M, Rhodes C J, Moncol J I M and Mazur M 2006 Free radicals, metals and antioxidants in oxidative stress-induced cancer Chem. Biol. Interact. 1601

14. Dreher D and Junod A F 1996 Role of oxygen free radicals in cancer development Eur. J. Cancer. 32A 30

15. Singal P K, Khaper N, Palace V and Kumar D 1998 The role of oxidative stress in the genesis of heart disease Cardiovasc. Res. 40426

16. Maneesh M, Jayalekshmi H, Suma T, Chatterjee S, Chakrabarti A and Singh T A 2005 Evidence for 
oxidative stress in osteoarthritis Indian J. Clin. Biochem. 20129

17. Lepetsos P, Athanasios G and Papavassiliou 2016 ROS/oxidative stress signaling in osteoarthritis Biochem. Biophys. Acta $\mathbf{1 8 6 2} 576$

18. Cherubini A, Ruggiero C, Polidori M C and Mecocci P 2005 Potential markers of oxidative stress in stroke Free Radic. Biol. Med. 39 841

19. Bowler R P and Crapo J D 2002 Oxidative stress in allergic respiratory disease J. Allergy Clinic. Immunol. 110 349

20. Knight J A 2000 Review: Free radicals, antioxidants, and the immune system Ann. Clin. Lab. Sci. 30 145

21. Fahn S and Cohen G 1992 The oxidant stress hypothesis in Parkinson's disease: Evidence supporting it Ann. Neurol. 32804

22. Tudr R M, Zhen L, Cho C Y, Taraseviciene-Stewart L, Kasahara Y, Salvemini D, Voelkel N F and Flores S C 2003 Oxidative stress and apoptosis interact and cause emphysema due to vascular endothelial growth factor receptor blockade Am. J. Respir. Cell Mol. Biol. 29 88

23. Adegboye A A, Khan K M, Salar U, Aboaba S A, Kanwal, Chigurapati S, Fatima I, Taha M, Wadood A, Mohammad J I, Khan H and Perveen S 2018 2-Aryl benzimidazoles: Synthesis, In vitro a-amylase inhibitory activity, and molecular docking study Eur. J. Med. Chem. 150248

24. Taha M, Imran S, Ismakil N H, Selvaraj M, Rashim F, Chigurapati S, Ullah H, Khan F, Salar U, Javid M T, Vijayabalan S, Zaman K and Khan K M 2017 Biologyoriented drug synthesis (BIODS) of 2-(2-methyl-5-nitro$1 \mathrm{H}$-imidazol-1-yl)ethyl aryl ether derivatives, in vitro $\alpha$ amylase inhibitory activity and in silico studies Bioorg. Chem. 741
25. Walker K A M, Wallach M B and Hirchfeld D R 1981 1-(Naphthylalkyl)-1H-imidazole derivatives, a new class of anticonvulsant agents J. Med. Chem. 2467

26. Karakurt A, Dalkara S, Ozalp M, Ozbey S, Kendi E and Stables J P 2001 Synthesis of some 1-(2-naphthyl)-2(imidazole-1-yl)ethanone oxime and oxime ether derivatives and their anticonvulsant and antimicrobial activities Eur. J. Med. Chem. 36421

27. Rokade Y B and Sayyed R Z 2009 Naphthalene derivatives: A new range of antimicrobials with high therapeutic value Rasayan J. Chem. 2972

28. Azarifar D and Shaebanzaadeh M 2002 Synthesis and characterization of new 3,5-dinaphthyl substituted 2pyrazolines and study of their antimicrobial activity Molecules 7885

29. Zhao G, Yin F-J, Ge H-Y and Li S-A 2014 Synthesis, structure and antibacterial properties of bis-imidazolebis(naphthalene-1-yl-acetato) copper (II) Asian J. Med. Chem. 262550

30. Jelen M, Bavavea E I, Pappa M, Kourounakis A P, Morak-Mlodwaska B and Pluta K 2015 Synthesis of quinoline/naphthalene-containing azaphenothiazines and their potent in vitro antioxidant properties Med. Chem. Res. 241725

31. Tay F, Erkan C, Sariozlu N Y, Ergene E and Demirayak S 2017 Synthesis, antimicrobial and anticancer activities of some naphthylthiazolylamine derivatives Biomed. Res. 282696

32. Ali I, Lone M N and Aboul-Enein H Y 2017 Imidazoles as potential anticancer agents Med. Chem. Commun. 8 1742

33. Miller G L 1959 Use of dinitrosalicylic acid reagent for determination of reducing Sugar Anal. Chem. 31426

34. Kumar S, Kumar D, Manjusha, Saroha K, Singh N and Vashishta B 2008 Antioxidant and free radical scavenging potential of Citrullus colocynthis (L.) Schrad. methanolic fruit extract Acta Pharm. 58215 